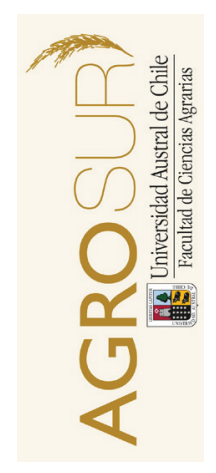

\title{
Efecto de la densidad de pastoreo sobre la capacidad de soporte y funcionalidad del sistema poroso de un Andisol
}

\author{
Effect of grazing density on the bearing capacity and functionality \\ of the pore system of an Andisol
}

\author{
Navarro, G. ${ }^{a}$, Dec, D. ${ }^{a, b}$, Balocchi, O. ${ }^{c}$, López, I. ${ }^{d}$, Clunes, J. ${ }^{a}$, Dörner, J. ${ }^{a, b *}$ \\ ${ }^{a}$ Instituto de Ingeniería Agraria y Suelos, Universidad Austral de Chile. Casilla 567, Valdivia, Chile. \\ ${ }^{b}$ Centro de Investigación en Suelos Volcánicos, Universidad Austral de Chile. Casilla 567, Valdivia, Chile. \\ ${ }^{c}$ Instituto de Producción Animal, Universidad Austral de Chile. Casilla 567, Valdivia, Chile. \\ ${ }^{d}$ Institute of Agriculture and Environment, Massey University, Palmerston North, New Zealand.
}

\begin{tabular}{l} 
A R T I C L E IN F O \\
\hline Article history: \\
Received 24.03.2017 \\
Accepted 06.03.2018 \\
\hline Keywords: \\
Static load \\
Dinamyc load \\
Volcanic soil \\
Grazing intensity \\
Resilience \\
\hline
\end{tabular}

Original Research Article,

Soil Science

${ }^{*}$ Corresponding author:

José Dörner

E-mail address:

josedorner@uach.cl
A B S T R A C T

Grazing management is a dynamic process that affects both the plants and the soil that supports them. The objective of this work was to evaluate the effect of grazing density on the bearing capacity and the functionality of the porous system of a Duric Hapludand, analysing its recovery. Two grazing densities of 50 and 200 cows ha ${ }^{-1}$ were established on a natural pasture regenerated with Lolium perenne L. and Trifolium repens L. $\left(20 \times 20 \mathrm{~m}^{2}\right.$ plots). In each experimental unit, disturbed and undisturbed soil samples $(2$ to $10 \mathrm{~cm}$ ) were collected in different moments: 1 day before grazing, 1 day after the last grazing and after a lag period of 105 days without grazing. On the undisturbed and packed samples at the same bulk density, the bearing capacity (CS, at $6 \mathrm{kPa}$ of tension) was determined with static loading test (CS_est) and cyclic loading test (CS_din). Additionally, the bulk density (da), air capacity (AC), air conductivity (Kl) and coefficient of linear extensibility (COLE) were determined. It was observed that continuous grazing affects the mechanical stability of the soil and the functionality of its pores. An increase in the grazing density during winter increases the CS value of the soil, being higher when calculated with CS_din. However, this relationship was not observed when analysing soil resilience. There were no critical values for the development of plant roots; however, it does not mean that these soils can endure stresses indefinitely.

\section{RESUMEN}

El manejo del pastoreo es un proceso dinámico que afecta tanto a la planta como al suelo que las sustenta. El objetivo de este trabajo fue evaluar el efecto de la densidad de pastoreo sobre la capacidad de soporte y la funcionalidad del sistema poroso de un Duric Hapludand. Se establecieron 2 densidades de pastoreo de 50 y 200 vacas ha ${ }^{-1}$ sobre una pradera natural regenerada con Lolium perenne L. y Trifolium repens L. (parcelas de 20x20 $\mathrm{m}^{2}$ ). Se colectaron muestras disturbadas y no disturbadas de suelo $(2 \mathrm{a} 10 \mathrm{~cm}$ ) en diferentes momentos: 1 día antes de los pastoreos, 1 día después del último pastoreo y luego de un periodo de rezago de 105 días sin pastoreo. En las muestras estructuradas y ensambladas a la misma densidad aparente, se determinó la capacidad de soporte (CS, a $6 \mathrm{kPa}$ de tensión) con cargas estáticas (CS_est) y dinámicas (CS_din). Además, se calculó la densidad aparente (da), capacidad de aire (CA), conductividad de aire (KI) y coeficiente de extensibilidad lineal (COLE). Se observó que los pastoreos continuos afectan la estabilidad mecánica del suelo y la funcionalidad de sus poros. Al aumentar la densidad de pastoreo durante el invierno se incrementa el valor de CS del suelo, siendo mayor cuando se calcula con CS_din. Sin embargo, esta relación no se repite al analizar la resilencia del suelo. No se presentaron valores críticos para el desarrollo de las raíces de las plantas, sin embargo, esto no significa que estos suelos puedan soportar estreses indefinidamente.

Palabras clave: cargas estáticas, cargas dinámicas, suelo volcánico, intensidad del pastoreo, resiliencia.

\section{INTRODUCCIÓN}

El pastoreo o la obtención in situ del alimento por parte del animal (Hodgson y Illius, 1996) es un fenómeno dinámico, existiendo una constante interacción entre este, la planta y el suelo que la sustenta (Greenwood y McKenzie, 2001; Bañuelos et al., 2007). Esta interacción modifica el crecimiento de las praderas debido a la defoliación, deposiciones y tránsito del animal durante el pastoreo (Menneer et al., 2004; Teuber et al., 2007). El tránsito continuo provoca la compactación del suelo, lo que implica un aumento en su densidad aparente y una 
disminución en el tamaño y la continuidad de los poros, afectando su capacidad para almacenar y transmitir agua y fluidos (Krümmelbein et al., 2008; Reszkowska et al., 2011a; Dec et al., 2011, 2012). La magnitud de estos cambios en el sistema poroso varía según la intensidad y duración de los eventos de pastoreo, la raza del animal que pastorea (Zhao et al., 2007; WingchingJones et al., 2009) y el contenido de agua en el suelo al momento del pastoreo (Dec et al., 2011, 2012).

$\mathrm{Al}$ pastorear con altos contenidos de agua se produce una deformación irreversible del suelo, lo que aumenta los riesgos de erosión por escurrimiento superficial (Drewry, 2006; Dec et al., 2011). Uno de los parámetros más utilizados para describir el comportamiento del suelo ante una carga y su resistencia a ser deformado es la capacidad de soporte (CS) (Tobias y Tietje, 2007; Horn y Fleige, 2009). En ese contexto, si su valor es menor a la presión ejercida por las pezuñas del animal en pastoreo (Krümmelbein et al., 2008), el suelo reaccionará plásticamente, perdiendo permanentemente sus características iniciales (Keller et al., 2004; Tomas et al., 2007). Sin embargo, al mismo tiempo el suelo tiene la capacidad de recuperarse después de una deformación, propiedad definida como resiliencia (Lal, 1994; Dörner et al., 2011), la que depende de las propiedades inherentes del suelo, su uso y el clima (asociado a ciclos de humectación y secado del suelo). Por lo tanto, el suelo es un sistema dinámico expuesto a cambios en su sistema poroso por acción de fuerzas externas e internas.

La CS es un buen parámetro para cuantificar la resistencia mecánica del suelo, sin embargo, su cálculo no considera el hecho que en ecosistemas pratenses las cargas aplicadas por los animales son de naturaleza cíclica o dinámica (Reszkowska et al., 2011a), o sea, se caracterizan por presentar ciclos repetitivos de carga y descarga a lo largo del año. Este proceso lleva a una alta deformación del suelo, destrucción de su estructura, cambios marcados en la distribución de su porosidad y disminución de su funcionalidad (Horn et al., 2003; Schäffer et al., 2007).

Existen estudios que han evaluado el efecto de estos ciclos sobre el sistema poroso de distintos suelos (Peth y Horn 2006; Krümmelbein et al., 2008; Reszkowska et al., 2011a); sin embargo, hay poca información de la evaluación de dichos efectos en suelos derivados de cenizas volcánicas (Ivelic-Sáez et al., 2015). Lo anterior es relevante, ya que los sistemas de producción ganadera en la zona sur de Chile se basan en el pastoreo constante de los animales sobre la pradera (Teuber et al., 2007), lo que expone al suelo a ciclos de compresión y recuperación. El objetivo de este estudio fue evaluar el efecto del pastoreo invernal (dos densidades de pastoreo) y un posterior periodo de rezago sobre la CS y funcionalidad del sistema poroso de un suelo derivado de cenizas volcánicas del sur de Chile.

\section{MATERIAL Y MÉTODOS}

\section{Suelo, historial de manejo y tratamientos}

El ensayo se llevó a cabo en la Estación Experimental Agropecuaria Austral (EEAA) (Valdivia, Chile. $39^{\circ} 46^{\prime}$ S, 73¹3' E; 12 m.s.n.m), sobre un suelo derivado de cenizas volcánicas de la Serie Valdivia, clasificado como Duric Hapludand (CIREN, 2003). La zona del ensayo se caracteriza por clima templado lluvioso de influencia marina, con una temperatura promedio de $12{ }^{\circ} \mathrm{C}$ y precipitaciones del orden de $2500 \mathrm{~mm}$ por año (GonzálezReyes y Muñoz, 2013).

El experimento seestableció en 6 parcelas $\left(20 \times 20 \mathrm{~m}^{2}\right)$ distribuidas al azar, lo que permitió generar dos tratamientos con tres repeticiones en un área total de $2400 \mathrm{~m}^{2}$. En abril de 2008 se sembró Lolium perenne L. (70\%) y Trifolium repens L. (30\%) sobre una pradera naturalizada de 15 años de antigüedad. Los tratamientos fueron de 200 vacas ha $^{-1}$ (A) y 50 vacas ha-1 (B), utilizando hembras adultas de raza Holstein Friesian $\left(\sim 500 \mathrm{~kg}\right.$ animal $\left.{ }^{-1}\right)$. Los eventos de pastoreos se relacionaron a la disponibilidad de forraje de la pradera, comenzando con 1700-2400 kg de materia seca (MS) y terminando con 1200-1500 kg MS según temporada; invierno y verano, respectivamente.

Se colectaron muestras disturbadas y no disturbadas de suelo $(2 \mathrm{a} 10 \mathrm{~cm})$ durante la temporada de invierno-primavera del segundo año del establecimiento del experimento. Esta labor se realizó 1 día antes de los pastoreos invernales (A_Past; 13.07.2010), 1 día después del último pastoreo invernal (D_Past; 05.09.2010) y luego de un periodo de rezago de 105 días sin pastoreo (Rec; 20.12.2010) para cada tratamiento y repetición (Cuadro 1). Durante el experimento se llevaron a cabo dos eventos de pastoreo $(14.07 .2010 \mathrm{y}$ 04.09.2010), dejando los animales en las unidades experimentales por un periodo de tiempo definido por la disponibilidad de forraje de la pradera, comenzando con 1700-2400 kg de materia seca (MS) y terminando con 1200-1500 kg MS según fuese invierno o verano. Las muestras no disturbadas se tomaron en cilindros metálicos (vol: $115 \mathrm{~cm}^{3}$; h: $3 \mathrm{~cm}$; d: $3,5 \mathrm{~cm}$ ) para medir variables mecánicas, como CS, aplicando cargas estáticas y dinámicas, que permiten simular la pisada del animal en condiciones de campo. Una vez que las muestras fueron tomadas, se cubrieron para evitar alteraciones mecánicas y evaporación.

\section{Análisis de laboratorio}

\section{Ensayo de consolidación mediante cargas estáticas y dinámicas}

Con el fin de determinar el efecto de la estructura sobre las variables estudiadas se prepararon cilindros 
Cuadro 1. Tratamientos aplicados, donde A y B son alta y baja densidad de pastoreo, respectivamente.

Table 1. Applied treatments, where A and B are high and low grazing density, respectively.

\begin{tabular}{|c|c|c|c|}
\hline Tratamiento & Densidad de pastoreo & Tipo de suelo & Carga aplicada \\
\hline Trat. 1 & $A\left(200 \mathrm{a} \mathrm{ha}^{-1}\right)$ & Estructurado & Estática \\
\hline Trat. 2 & $\mathrm{~B}\left(50 \mathrm{a} \mathrm{ha}^{-1}\right)$ & Estructurado & Estática \\
\hline Trat. 3 & $A\left(200 \mathrm{a} \mathrm{ha}^{-1}\right)$ & Estructurado & Dinámica \\
\hline Trat. 4 & $\mathrm{~B}\left(50 \mathrm{a} \mathrm{ha}^{-1}\right)$ & Estructurado & Dinámica \\
\hline Trat. 5 & $\mathrm{~A}\left(200 \mathrm{a} \mathrm{ha}^{-1}\right)$ & Homogenizado & Estática \\
\hline Trat. 6 & $\mathrm{~B}\left(50 \mathrm{a} \mathrm{ha}^{-1}\right)$ & Homogenizado & Estática \\
\hline Trat. 7 & $\mathrm{~A}\left(200 \mathrm{a} \mathrm{ha}^{-1}\right)$ & Homogenizado & Dinámica \\
\hline Trat. 8 & $\mathrm{~B}\left(50 \mathrm{a} \mathrm{ha}^{-1}\right)$ & Homogenizado & Dinámica \\
\hline
\end{tabular}

Cada tratamiento considera los valores de muestreo a: A_Past (antes de los pastoreos invernales); D_Past (después de los pastoreos invernales) y Rec (recuperado; luego de 105 días sin pastoreo).

a partir del material disturbado (suelo homogenizado), ajustando la densidad aparente (da) y el contenido de agua correspondiente a cada cilindro recolectado a nivel de campo. Para obtener la da deseada se siguió la metodología descrita por Demond et al. (1996) y utilizada por Dörner et al. (2010), la cual consiste en tomar el suelo tamizado a $2 \mathrm{~mm}$, seco a $30^{\circ} \mathrm{C}$ y rehumectado, igualando la da y el contenido de humedad de la muestra original. El suelo se coloca en el cilindro metálico separando en 4 estratas iguales donde cada capa de suelo se comprime suavemente al espacio que le corresponde por sección de cilindro. Todas las muestras fueron saturadas por capilaridad y equilibradas a $6 \mathrm{kPa}$ de tensión previo a los ensayos de consolidación.

Para determinar la capacidad de soporte a partir de cargas estáticas (CS_est) se calculó la curva de consolidación del suelo, utilizando un odómetro con drenaje (Controls T303) y aplicando cargas sucesivas de 6,25 - 12,5 - 25 - 50 - 100 - 200 y $400 \mathrm{kPa}$ de 6 minutos cada una y la posterior descarga de $200-100-50$ y $6,25 \mathrm{kPa}$ de 6 minutos cada una, durante los cuales se midió la deformación vertical (precisión: 0,01 mm). La capacidad de soporte con cargas dinámicas (CS_din) se determinó realizando un test de cargas cíclicas progresivas (Peth y Horn, 2006; Reszkowska et al., 2011a), aplicando ciclos repetitivos de carga y descarga sobre las muestras de suelo y midiendo la deformación de las muestras en 10 ciclos de $30 \mathrm{~s}$ de carga y $30 \mathrm{~s}$ de descarga para las siguientes presiones: $20-40$ - 50 - 75 - 100 - 150 - 200 - $400 \mathrm{kPa}$. Para ejecutar este ensayo se utilizó un odómetro de pistón modificado (Peth y Horn, 2006). La capacidad de soporte se calculó a partir de la curva de consolidación de las muestras de suelo según Casagrande (1936), utilizando como referencia la medida de deformación del último ciclo de carga para las distintas presiones aplicadas.

\section{Cálculo de índices para evaluar el comportamiento del sistema poroso del suelo}

Se calculó la densidad aparente (da) y la capacidad de aire (CA) de cada muestra analizada, como índices que permiten evaluar el comportamiento del sistema poroso del suelo. Para esto se obtuvo la relación entre la masa de la muestra de suelo seca a $105^{\circ} \mathrm{C}$ por $24 \mathrm{hrs}$ y el volumen del cilindro utilizado, en tanto la diferencia entre la porosidad total de la muestra y el contenido volumétrico de agua a $6 \mathrm{kPa}$ de tensión corresponde a la CA (Dörner et al., 2015). Para determinar el efecto de la intensidad de pastoreo sobre la funcionalidad de los poros se determinó la conductividad de aire del suelo (Kl) utilizando un flujómetro de aire (Key Instruments, Trevore, USA) mediante el método de flujo constante (Dörner y Horn, 2006). Esta propiedad se determinó previo (con muestras equilibradas a $-6 \mathrm{kPa}$ ) y luego del ensayo de consolidación. Para su cálculo se determinó la permeabilidad de aire $(K a)$ a través de la siguiente ecuación:

$$
K a\left(\varepsilon_{a}\right)=\mathrm{Kl}\left(\varepsilon_{a}\right) * \frac{\eta}{\rho_{l} * g}
$$

Donde: $K a$ : permeabilidad de aire $\left(\mu \mathrm{m}^{2}\right)$; Kl: conductividad de aire ( $\left.\mathrm{cm} \mathrm{s}^{-1}\right)$; $\varepsilon_{a}$ : volumen de poros con aire $\left(\mathrm{cm}^{3} \mathrm{~cm}^{-3}\right) ; \eta$ : viscosidad de aire $\left(\mathrm{g} \mathrm{s}^{-1} \mathrm{~cm}^{-1}\right) ; \rho_{l}$ : densidad de aire $\left(\mathrm{kg} \mathrm{m}^{-3}\right) ; g$ : aceleración de gravedad $\left(\mathrm{m} \mathrm{s}^{-2}\right)$. Tanto la viscosidad de aire como su densidad fueron corregidas según temperatura $\left({ }^{\circ} \mathrm{C}\right)$.

El coeficiente de extensibilidad lineal (COLE), que define la variación unidimensional del suelo desde su punto de saturación a condiciones de menor humedad, ha sido utilizado por Peng et al. (2007) y Dörner et al. (2009; 2011) para evaluar la deformación y resiliencia 
del suelo. Este índice, se determinó con el fin de evaluar el efecto de las distintas densidades de pastoreo sobre la deformación del suelo previo y posterior al proceso de consolidación. El COLE fue calculado de la siguiente manera:

$$
\operatorname{COLE}=\frac{L_{a}-L_{b}}{L_{105^{\circ} \mathrm{C}}}
$$

Donde $L_{a}$ : altura inicial del suelo $(0 \mathrm{kPa}$ de tensión, $\mathrm{mm}) ; L_{b}$ : altura del suelo en un momento determinado ( $6 \mathrm{kPa}$ de tensión y después de consolidación, $\mathrm{mm}$ ) y $L_{105^{\circ} \mathrm{C}}$ : altura de la muestra secada a $105^{\circ} \mathrm{C}$ por $24 \mathrm{hrs}$ $(\mathrm{mm})$.

\section{Análisis estadístico}

Para cada tratamiento y muestreo (Cuadro 1) se calcularon promedios y errores estándar. Parámetros que no presentaron una distribución normal se presentan en diagramas de caja, mostrando el valor medio, sus percentiles, mediana y su desviación estándar. Se realizó un análisis de varianza (ANDEVA) multifactorial para determinar el efecto de los tratamientos (alta y baja carga), tipo de muestra (estructurada, homogenizada), tipo de test (estático, dinámico) y los distintos muestreos (antes y después del pastoreo, después del rezago) sobre la CS del suelo y la funcionalidad de sus poros; en caso de comprobarse algún efecto significativo se aplicó un test LSD $(\mathrm{p}<0,01)$ para analizar diferencias en las medias. Además, se desarrollaron regresiones lineales, las cuales fueron determinadas para relacionar variables respuesta.

\section{RESULTADOS Y DISCUSIÓN}

\section{Efecto de la intensidad de pastoreo sobre la CS del suelo}

Los valores de capacidad de soporte del suelo antes de los pastoreos de invierno (A_Past) se ubican en el rango de 12,1 como mínimo a 44,0 kPa como máximo (suelo estructurado media de $37,3 \mathrm{kPa}$; CV: $25 \%$; suelo homogenizado media de 19,8 kPa; CV: 44\%); después de los pastoreos en la misma estación (D_Past) se registraron valores de 15,3 a 59,2 kPa (suelo estructurado media de 48,6 $\mathrm{kPa}$; CV: $30 \%$; suelo homogenizado media de 28,4 kPa; CV: 36\%) y 105 días después del último pastoreo de invierno (Rec) de 13,9 a $50,8 \mathrm{kPa}$ (suelo estructurado media de $42,5 \mathrm{kPa}$; $\mathrm{CV}$ : $26 \%$; suelo homogenizado media de $25,2 \mathrm{kPa}$; $\mathrm{CV}$ : $40 \%$ ). Además, el análisis estadístico realizado indica que existe una interacción positiva entre las variables estudiadas $(p<0,05)$, indicando que el efecto de los tratamientos sobre la CS del suelo varía de acuerdo con los muestreos realizados (Figura 1A).
La CS es significativamente menor en suelos que han perdido su estructura, para todos los tratamientos y muestreos, aun cuando suelos estructurado v/s homogenizados tienen los mismos valores de densidad aparente (aprox. 0,7 $\mathrm{Mg} \mathrm{m}^{-3}$ ) (Cuadro 3). Estos resultados coinciden con los expuestos por Peth y Horn (2006) y Reszkowska et al. (2011a) para suelos bajo pastoreo animal en Alemania y Mongolia, respectivamente. Los autores indican que la CS calculada de manera estática y dinámica es significativamente menor en suelos homogenizados, donde las partículas del suelo están débilmente cohesionadas. A su vez, la materia orgánica (MO) y las raíces de las plantas son fundamentales para el desarrollo de poros más estables en el suelo, las cuales se pierden en el proceso de homogenización de las muestras (Horn y Smucker, 2005). Por lo tanto, una pérdida de estructura producida por la labranza del suelo, reduce significativamente su CS (Dörner et al., 2012). Sin embargo, el suelo como cuerpo dinámico está en constante proceso de formación de estructura (Vásquez et al., 2012), que permite recuperar los valores originales de su resistencia mecánica.

La CS del suelo calculada con cargas dinámicas $\left(C_{-}\right.$din $)$es significativamente mayor que la calculada con cargas estáticas ( $\left.C_{-} e s t\right)$, para todos los tratamientos y muestreos realizados, existiendo una correlación positiva entre $C S_{-}$din y $C S_{-}$est (Cuadro 2); $\mathrm{A}_{-}$Past $\left(\mathrm{R}^{2}=0,78\right.$; $P>0,05)$, D_Past $\left(R^{2}=0,98 ; P<0,01\right)$ y $\operatorname{Rec}\left(R^{2}=0,95\right.$; $\mathrm{P}<0,05$ ) (Figura 1A). Estas relaciones son concordantes con las obtenidas por Krümmelbein et al. (2008) y

Cuadro 2. Capacidad de soporte (CS, kPa) del suelo en función del tratamiento.

Table 2. Soil bearing capacity (CS, $\mathrm{kPa})$ as function of treatment.

\begin{tabular}{llc}
\hline Tratamiento & Descripción & $\begin{array}{c}\text { Capacidad de Soporte } \\
\text { (CS) [kPa] }\end{array}$ \\
\hline Trat. 1 & A / EST / C_est & $34,17 \pm 1,37$ c \\
Trat. 2 & B / EST / C_est & $29,47 \pm 1,00$ d \\
Trat. 3 & A / EST / C_din & $51,33 \pm 1,35$ a \\
Trat. 4 & B / EST / C_din & $44,59 \pm 1,25$ b \\
Trat. 5 & A / HOM / C_est & $15,59 \pm 0,54$ e \\
Trat. 6 & B / HOM / C_est & $13,79 \pm 0,34$ e \\
Trat. 7 & A / HOM / C_din & $32,82 \pm 1,17$ cd \\
Trat. 8 & B / HOM / C_din & $25,92 \pm 0,83$ e \\
\hline
\end{tabular}

Se presentan valores promedio \pm error estándar. Letras minúsculas distintas indican diferencias entre tratamientos $(p \leq 0,01)$. A $(200$ vacas por ha-1); B (50 vacas por ha' ${ }^{-1}$ ). EST (muestra estructurada); HOM (muestra homogenizada). C_est (cargas estáticas); C_din (cargas dinámicas). 
Reszkowska et al. (2011a) en suelos de Mongolia (Calcic Chernozem) bajo pastoreo ovino. Estos autores indican que la aplicación de repetidas cargas sobre el suelo causa una mayor deformación que la aplicación de cargas estáticas. Además, se ha determinado que cargas repetitivas deforman plásticamente el suelo, incluso si no es superado su valor de preconsolidación (Peth y Horn, 2006). Lo anterior está asociado a una pequeña deformación acumulativa producida por la aplicación de ciclos de carga y descarga constantes en el tiempo, aunque estas cargas aplicadas sean pequeñas en comparación a su capacidad de soporte (Horn et al., 2003; Wichtmann et al., 2005). Esta deformación acumulativa, depende directamente de la intensidad del pastoreo animal (Krümmelbein et al., 2008) o el número de ciclos aplicados sobre el suelo (Peth y Horn, 2006).

Al aumentar la intensidad de pastoreo (de 50 a 200 vacas ha-1) para un mismo tipo de suelo y carga aplicada, se observa una mayor resistencia mecánica a la deformación en los tratamientos con mayor carga animal, aunque no significativa para el tratamiento de muestra homogenizada con cargas estáticas (HOM/C_est). En este contexto, los valores de CS no reflejaron problemas severos de compactación (Cuadro 2), ya que valores de CS mayores a $90 \mathrm{kPa}$ son restrictivos para el desarrollo de las raíces (Horn y Fleige, 2009). Tomando en cuenta estos resultados, se puede concluir que, a pesar del aumento de la resistencia mecánica del suelo derivado de cenizas volcánicas debido al tránsito animal, no se llegó a valores restrictivos para el crecimiento de las raíces, confirmando la alta estabilidad mecánica determinada por Dec et al. (2012) para este tipo de suelo. Lo anterior se debe a las características intrínsecas de los Andisoles, que reducen los impactos del pastoreo ani- mal sobre la estabilidad mecánica del suelo, debido a los altos contenidos de materia orgánica (complejos AlMO) en horizontes superficiales (Matus et al., 2006), lo que le confiere elasticidad y resiliencia al suelo (Ellies, 1988; Ivelic-Sáez et al., 2015). Junto con lo anterior, la alta estabilidad al agua, fuerte microagregación de formas heterogéneas (Ellies et al., 1997; Hoyos y Comerford, 2005), alta capacidad de contracción (Bartoli et al., 2007; Dörner et al., 2009) y una gran conductividad hidráulica, permiten sustentar el pastoreo sin que se produzca un detrimento significativo en los poros del suelo. Sin embargo, la estabilidad mecánica del suelo varía en el corto plazo, dependiendo del contenido de agua, los eventos de pastoreo y su intensidad (Horn y Baumgartl, 2002; Dec et al., 2011, 2012).

El pastoreo durante el invierno aumenta significativamente la CS $(34,90 \pm 1,32 \mathrm{kPa})$ como resultado de la deformación (Krümmelbein et al., 2008), la estabilización mecánica y la compactación del suelo (Peth y Horn, 2006) que producen las pezuñas del animal por su tránsito en condiciones de alta humedad (menor cohesión entre agredados y partículas del suelo). Después de dos meses de rezago y cese del pastoreo (Rec) el suelo disminuye su CS, sin embrago, no alcanza los niveles iniciales (Figura 1A). Los cambios en la estructura de los Andisoles producto de pastoreos continuos (e.g. la capacidad de aire del suelo) pueden recuperarse cuando su frecuencia disminuye (Dec et al., 2012), dependiendo directamente de la relación entre las precipitaciones, la temperatura (induciendo ciclos de humectación y secado) y la actividad microbiológica del suelo (Horn y Smucker, 2005) que en conjunto le otorgan a estos suelos una alta resiliencia (Dörner et al., 2009; 2011).

Cuadro 3. Densidad aparente (da) y capacidad de aire (CA) del suelo en función del tratamiento.

Table 3. Bulk density (da) and air capacity (CA) of the soil as a function of treatment.

\begin{tabular}{|c|c|c|c|}
\hline Tratamiento & Descripción & Densidad aparente (da) $\left[\mathrm{Mg} \mathrm{m}^{-3}\right]$ & Capacidad de Aire (CA) [\%] \\
\hline Trat. 1 & A / EST / C_est & $0,68 \pm 0,00 a b$ & $12,96 \pm 0,63 b$ \\
\hline Trat. 2 & B / EST / C_est & $0,69 \pm 0,00 \quad a$ & $14,03 \pm 0,66 b$ \\
\hline Trat. 3 & A / EST / $C_{-}$din & $0,67 \pm 0,00 \quad a b$ & $12,17 \pm 0,48 \quad b$ \\
\hline Trat. 4 & $\mathrm{~B} / \mathrm{EST} / C_{-} d i n$ & $0,68 \pm 0,00 \mathrm{ab}$ & $13,09 \pm 0,36 b$ \\
\hline Trat. 5 & A / HOM / C_est & $0,68 \pm 0,01 a b$ & $17,87 \pm 1,02 a$ \\
\hline Trat. 6 & B / HOM / C_est & $0,67 \pm 0,00 \quad b$ & $17,04 \pm 0,49$ a \\
\hline Trat. 7 & $\mathrm{~A} / \mathrm{HOM} / C_{-} d i n$ & $0,66 \pm 0,00 \quad b$ & $18,37 \pm 0,53 a$ \\
\hline Trat. 8 & B / HOM / C_din & $0,66 \pm 0,00 \quad b$ & $17,46 \pm 0,43$ a \\
\hline
\end{tabular}

Se presentan valores promedio \pm error estándar. Letras minúsculas distintas indican diferencias entre tratamientos o muestreos $(\mathrm{p} \leq 0,01)$. A (200 vacas por ha $\left.{ }^{-1}\right)$; B (50 vacas por ha ${ }^{-1}$ ). EST (muestra estructurada); HOM (muestra homogenizada). $C_{-}$est (cargas estáticas); $C_{-}$din (cargas dinámicas). 


\section{Efecto del pastoreo sobre las funciones de los poros del suelo}

La densidad aparente (da) varía tanto con la aplicación de los distintos tratamientos (Cuadro 3) como con los muestreos realizados (Figura 1B), variando entre 0,67 a $0,69 \mathrm{Mg} \mathrm{m}^{-3}$, valores considerados normales para suelos derivados de cenizas volcánicas (FAO, 2006) y concordantes con los encontrados por Dörner et al. $(2011,2012)$ y Dec et al. (2012) para Andisoles. Greenwood et al. (1997) asocian el cambio en la da de un suelo bajo pastoreo sólo a horizontes superficiales, siendo los valores obtenidos significativamente menores a los considerados críticos para el crecimiento de las raíces de las plantas $\left(1,7 \mathrm{Mg} \mathrm{m}^{-3}\right)$. Sin embargo, la da no es considerada como el parámetro más adecuado para indicar pérdida de funciones del suelo producto de una compactación (Cuadro 3), ya que su cálculo relaciona masa en un volumen determinado, sin considerar el ordenamiento de estas partículas, que finalmente dan la habilidad para contener y transmitir agua y aire (Dec et al., 2011; Dörner et al., 2012).

Los valores de capacidad de aire (CA) variaron entre $11,7 \%$ y $20,7 \%$, existiendo una disminución significativa de la macroporosidad luego de los pastoreos invernales (Figura 1C) y una leve tendencia a aumentar luego de 105 días sin pastorear. Durante el periodo de estudio, la CA superó el valor considerado como límite crítico para un adecuado intercambio gaseoso (8\%), por lo tanto, no existirían condiciones que podrían restringuir el desarrollo de las plantas (Horn y Fleige, 2009). Esta situación se debe a la alta porosidad gruesa que poseen estos suelos, y si bien, el tránsito animal genera compactación, perdiéndose primeramente la macroporosidad (Fujikawa y Miyazaki, 2005), aún queda un remanente de poros gruesos, resultando finalmente todo este proceso en un aumento de la capacidad de soporte $\left(R^{2}=0,30 ; P>0,05\right)$.

Se ha determinado que el flujo de aire dentro del perfil de suelo no depende directamente de su CA (o macroporosidad), sino más bien, de la capacidad que tengan estos poros para conducir, lo que está dado por aspectos geométricos del espacio poroso, como su continuidad, interconexión y tortuosidad (Dörner y Horn, 2006). Al respecto, la conductividad de aire (Kl) es una variable adecuada para determinar la conectividad de los poros del suelo, así como su funcionalidad (Reszkowska et al., 2011a). Los valores de Kl durante el estudio, antes y después de la consolidación de las muestras, presentaron rangos entre $-5,3$ y $-4,7 \log \mathrm{m}$ $\mathrm{s}^{-1}$ y $-6,1$ y $-5,5 \log \mathrm{m} \mathrm{s}^{-1}$, respectivamente (Figura 2). Los cambios en los valores de $\mathrm{Kl}$ muestran el efecto de la compactación sobre la continuidad de sus poros (Dec et al., 2011). Por otra parte, existe una influencia negativa del pastoreo animal sobre $\mathrm{Kl}$ (Savadogo et al., 2007; Reszkowska et al., 2011b) reflejada en la dismi- nución de esta propiedad en el muestreo D_Past (no significativa). Pastoreos animales continuos promueven el bloqueo del sistema poroso del suelo (Drewry, 2006), disminuyendo el número de poros verticales,


Figura 1. Capacidad de soporte (A), densidad aparente (B) y capacidad de aire (C) del suelo en función del muestreo realizado. Se presentan valores promedio \pm error estándar. Letras minúsculas distintas indican diferencias entre muestreos ( $p \leq 0,01)$. A_Past (antes pastoreo inv.); D_Past (después pastoreo inv.); Rec (105 días luego de pastoreo inv.).

Figure 1. Bearing capacity (A), bulk density (B) and air capacity (C) of the soil as a function of sampling. Average values \pm standard errors are presented. Different lowercase letters indicate differences between samplings ( $p \leq 0.01$ ). A_Past (before winter grazing); D_Past (after winter grazing); Rec (105 days after winter grazing). 
lo que aumenta la proporción de poros horizontales, siendo la conductividad en este último sentido muy baja (Mosaddeghi et al., 2007; Reszkowska et al., 2011a). Luego de un periodo de rezago de pastoreo (105 días) se observa un leve aumento de Kl (no significativo), sin indicar este una tendencia clara del suelo a recuperar sus atributos funcionales, lo que es asociado principalmente al poco tiempo transcurrido entre muestreos. En este sentido, la estabilidad y resiliencia mecánica no se refleja necesariamente en la funcionalidad porosa.

No se determinó una correlación significativa entre la Kl y la CA (Figura 3), lo cual indica que a igual capacidad de aire en el suelo, este es capaz de conducir distintos montos de aire por convección, dependiendo esta propiedad de la interconexión y continuidad del sistema poroso más que de su monto (Dörner y Horn, 2006; Dörner et al., 2010). Valores similares fueron encontrados por Dec et al. (2012) para suelos derivados de cenizas volcánicas.
El coeficiente de extensibilidad lineal (COLE), que representa la deformación unidimensional del suelo desde saturación a condiciones de menor humedad (Peng et al., 2007), presentó valores que variaron entre 0,01 y 0,034 (entre saturación y $6 \mathrm{kPa}$ de tensión) y de 0,092 a 0,403 luego de consolidado el suelo (Cuadro 4). Esta diferencia fue esperada debido a la deformación vertical que produce la consolidación de las muestras de suelo. Por otro lado, el COLE es inversamente proporcional a la $C S\left(R^{2}=0,31 ; p<0,01\right)$ y la densidad aparente $\left(R^{2}=0,20 ; p<0,05\right)$, como así también presentó una relación positiva (aunque no muy marcada) con el volumen de macroporos $\left(R^{2}=0,15 ; p<0,05\right)$. El estrés mecánico durante el invierno (pastoreo animal) aumentó la da y disminuyó CA del suelo, aumentando su estabilidad estructural y disminuyendo el COLE (efecto no significativo), fenómeno que se observa posterior a los pastoreos invernales (Cuadro 4b). Después de 3 meses de exclusión de pastoreo, se observa una recuperación significativa del COLEac (Cuadro 4b), que

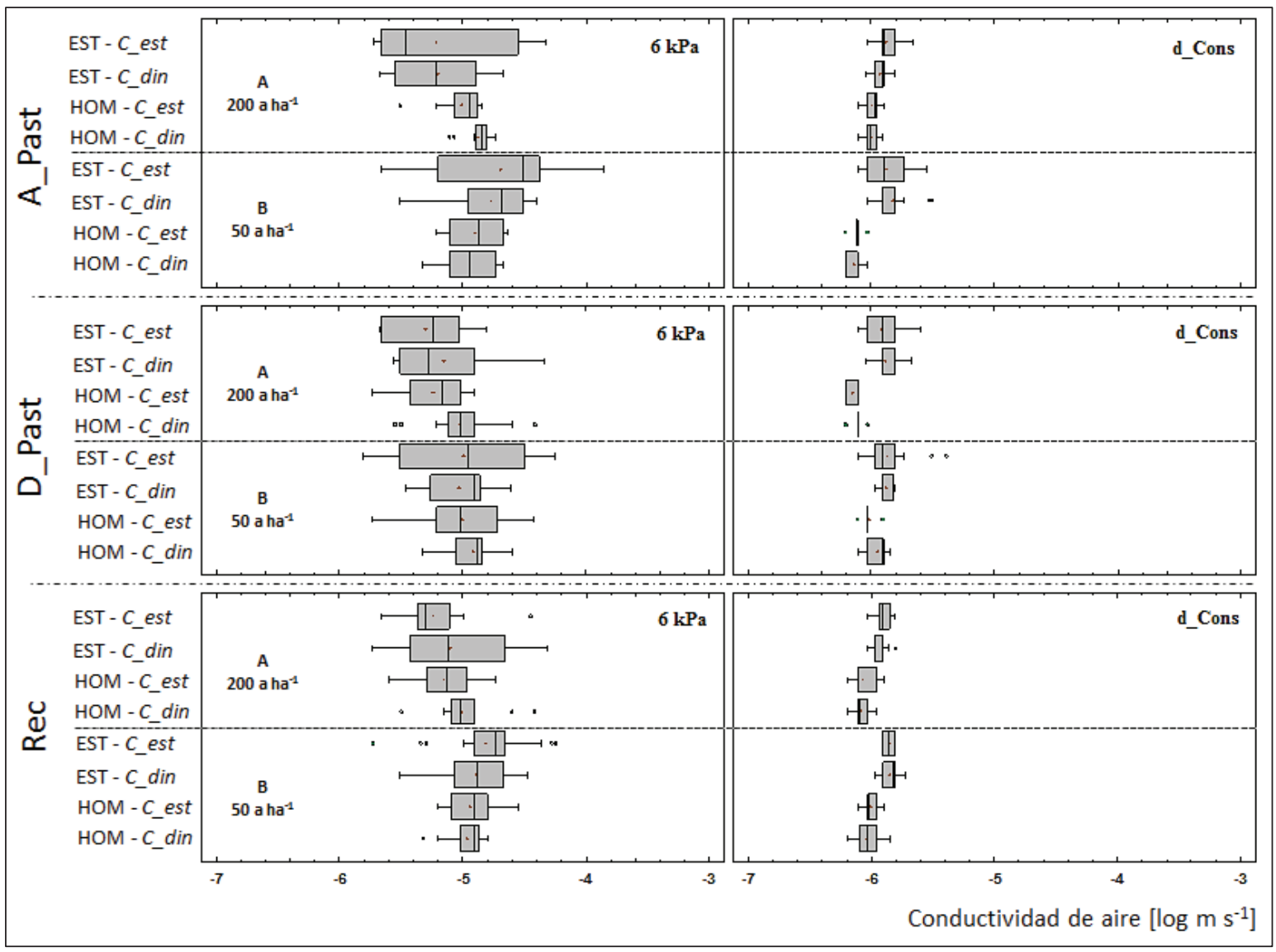

Figura 2. Conductividad de aire ( $\mathrm{Kl}$ ) antes (6 kPa de tensión) y después de la consolidación (d_Cons) en función de los tratamientos y muestreos aplicados. Diagrama de cajas presenta percentiles (P25 y P75), mediana, valor promedio y desviación estándar.

Figure 2. Air conductivity (Kl) at $6 \mathrm{kPa}$ of tension and after consolidation (d_Cons) as a function of treatments and samplings. Box diagram presents percentiles (P25 and P75), median, mean value and standard deviation. 


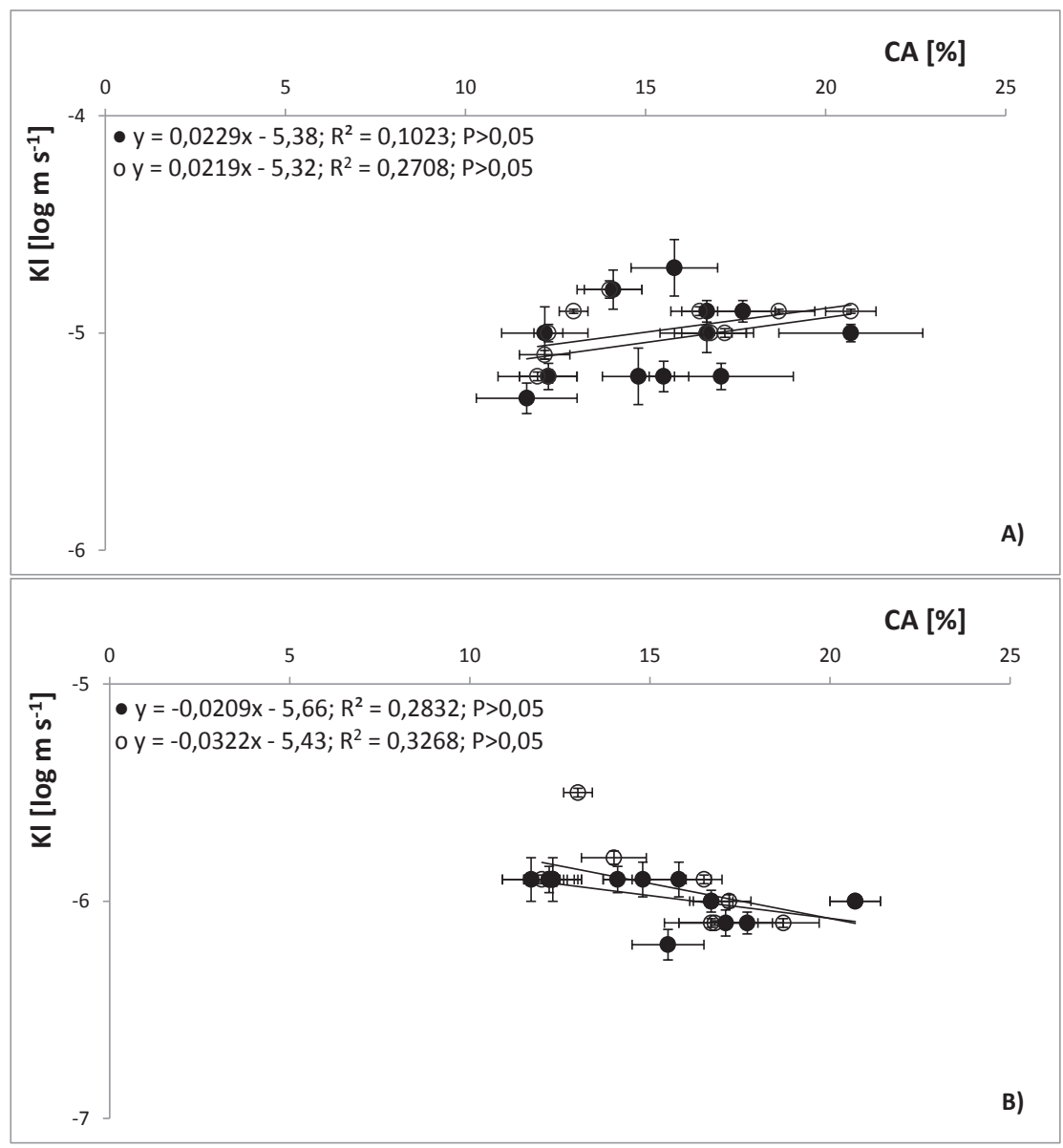

Figura 3. Conductividad de aire (Kl) en función de la capacidad de aire (CA) de las muestras. Kl a 6 kPa de tensión (A) y después de la consolidación (B). Consolidación con cargas estáticas (círculos negros); consolidación con cargas dinámicas (círculos blancos). Barras indican \pm 1 error estándar $(n=6)$.

Figure 3. Air conductivity (Kl) as function of air capacity (CA) of the samples. Kl at $6 \mathrm{kPa}$ of tension (A) and after consolidation (B). Consolidation with static loads (black circles); consolidation with dynamic loads (white circles). Bars indicate \pm 1 standard error $(n=6)$.

indica la gran elásticidad que presentan los Andisoles (Ellies, 1988; Dörner et al., 2011).

Un aspecto importante a la hora de explicar el comportamiento mecánico del suelo frente al pastoreo es la condición o tipo de pradera existente. Cualquier cambio en la pradera, como la siembra o la aplicación de estrategias para su mejoramiento, afectan la calidad física y los procesos internos del suelo en el corto y mediano plazo (Dörner et al., 2013). Además, el suelo bajo una pradera naturalizada pastoreada continuamente es mecánicamente más estable y posee una menor capacidad de contener y transmitir agua y aire que un suelo bajo una pradera sembrada y fertilizada al segundo año de medición (Dörner et al., 2013; Zúñiga et al., 2015). En el presente estudio, la pradera fue sembrada en abril de 2008 y hasta la fecha de toma de muestras (temporada invierno-primavera 2010) fueron cumplidos todos sus requerimientos de fertilización.
De esta manera, la pérdida de estructura del suelo por la labranza, en función de factores intrínsecos del suelo, factores ambientales, crecimiento acelerado de biomasa aérea y radicular producto de una adecuada fertilización y el manejo del pastoreo realizado, influyen en la formación y estabilización de la estructura del suelo (Dec et al., 2012). Tomando en cuenta los resultados, es necesario considerar la pérdida de atributos funcionales del suelo por el pastoreo como un problema que integre el complejo Suelo-Planta-Animal, siendo este la base para la implementación de nuevas metodologías que mitiguen o anulen los costos medioambientales de la producción animal.

\section{CONCLUSIONES}

Los pastoreos continuos durante la temporada de invierno afectan tanto la estabilidad mecánica del sue- 
Cuadro 4. Coeficiente de extensibilidad lineal (COLE) (entre 0 y $6 \mathrm{kPa}$ de tensión de agua) antes $(a c)$ y después $(d c)$ de la consolidación del suelo en función del tratamiento (a) y muestreo realizado (b).

Table 4. Coefficient of linear extensibility (COLE) (beween 0 and $6 \mathrm{kPa}$ of water tension) before $(a c)$ and after $(d c)$ soil consolidation as a function of treatment (a) and sampling (b).

a)

\begin{tabular}{|c|c|c|c|}
\hline Tratamiento & Descripción & Coef. Extensibilidad Lineal COLEac; [-] & Coef. Extensibilidad Lineal COLEdc; [-] \\
\hline Trat. 1 & $\mathrm{~A} / \mathrm{EST} / C_{-}$est & $0,0162 \pm 0,00 \mathrm{~d}$ & $0,1851 \pm 0,00 \mathrm{de}$ \\
\hline Trat. 2 & $\mathrm{~B} / \mathrm{EST} / C_{-}$est & $0,0205 \pm 0,00 \quad b c$ & $0,2136 \pm 0,00 \quad c$ \\
\hline Trat. 3 & A / EST / $C_{-}$din & $0,0112 \pm 0,00$ e & $0,1477 \pm 0,00 \mathrm{f}$ \\
\hline Trat. 4 & B / EST / $C_{-} d i n$ & $0,0164 \pm 0,00 \mathrm{~d}$ & $0,1697 \pm 0,00$ ef \\
\hline Trat. 5 & A / HOM / C_est & $0,0186 \pm 0,00 \mathrm{~cd}$ & $0,2685 \pm 0,01 \quad b$ \\
\hline Trat. 6 & B / HOM / C_est & $0,0253 \pm 0,00 \mathrm{ab}$ & $0,2914 \pm 0,01 \mathrm{a}$ \\
\hline Trat. 7 & $\mathrm{~A} / \mathrm{HOM} / C_{-} d i n$ & $0,0154 \pm 0,00 d$ & $0,2010 \pm 0,01 \mathrm{~cd}$ \\
\hline Trat. 8 & B / HOM / C_din & $0,0230 \pm 0,00 \mathrm{ab}$ & $0,2634 \pm 0,01 \quad b$ \\
\hline \multicolumn{4}{|l|}{ b) } \\
\hline Muestreo & Descripción & Coef. Extensibilidad Lineal COLEac; [-] & Coef. Extensibilidad Lineal COLEdc; [-] \\
\hline Muestreo 1 & A_Past & $0,0219 \pm 0,00 \quad a$ & $0,2714 \pm 0,00 \quad a$ \\
\hline Muestreo 2 & D_Past & $0,0129 \pm 0,00 \quad b$ & $0,1477 \pm 0,00 \quad c$ \\
\hline Muestreo 3 & $\operatorname{Rec}$ & $0,0201 \pm 0,00 \quad a$ & $0,2319 \pm 0,00 \quad b$ \\
\hline
\end{tabular}

Se presentan valores promedio \pm error estándar. Letras minúsculas distintas indican diferencias entre tratamientos o muestreos ( $\mathrm{p} \leq 0,01)$. A (200 vacas por ha ${ }^{-1}$ ); B (50 vacas por ha-1). EST (muestra estructurada); HOM (muestra homogenizada). C_est (cargas estáticas); $C_{-}$din (cargas dinámicas). A_Past (antes pastoreo inv.); D_Past (después pastoreo inv.); Rec (105 días luego de pastoreo inv.).

lo como la funcionalidad de sus poros, demostrando la variación en el corto plazo de las propiedades estructurales del suelo. El tránsito animal causa un incremento en el valor de capacidad de soporte (CS) del suelo, asociado a la compactación producida por las pezuñas del animal. Al aumentar la densidad de pastoreo (de 50 a 200 vacas ha $^{-1}$ ) durante el invierno se induce una mayor estabilización mecánica del suelo, lo que no se reflejó en aspectos funcionales de su sistema poroso. Aunque, los Andisoles presentan una alta resiliencia, no se observó una recuperación significativa de sus atributos perdidos por los pastoreos invernales, asociado principalmente al corto periodo de exclusión de pastoreo (105 días).

No se presentan valores críticos para el desarrollo de las raíces de las plantas luego de la estabilización mecánica producida en el suelo por pastoreos invernales, asociado principalmente a las características intrínsecas de los suelos derivados de cenizas volcánicas y la condición de la pradera estudiada. Lo anterior, bajo ningún punto de vista significa que estos suelos pueden soportar estreses indefinidamente, por lo que se recomiendan estudios del comportamiento del suelo bajo pastoreo continuo en el largo plazo y para distintas condiciones de una pradera; además de considerar la naturaleza cíclica de las cargas aplicadas al suelo al momento de recomendar pastoreos continuos en suelos de la zona sur de Chile.

\section{REFERENCIAS}

Bañuelos, R., Echavarría, F., Serna, A., 2007. Influencia del sistema de pastoreo con pequeños rumiantes en un agostadero del semiárido Zacatecano: II Cambios en el suelo. México. Técnica Pecuaria en México 45(2), 177-194.

Bartoli, F., Begib, J., Burtin, G., Schouller, E., 2007. Shrinkage of initially very wet soil blocks, cores and clods from a range of european Andosol horizons. European Journal of Soil Science 58(2), 378-392. https://doi.org/10.1111/ j.1365-2389.2006.00889.x

Casagrande, A., 1936. Characteristics of cohesive soils affecting the stability of slopes and Earth fills. Journal of the Boston Society of Civil Engineers 23, 13-32.

Centro de Información de Recursos Naturales (CIREN), 2003. Estudio agrológico X Región. Descripción de suelos, materiales y símbolos. Publicación $N^{\circ} 123$, Santigo, Chile.

Dec, D., Dörner, J., Balocchi, O., 2011. Temporal and spatial variability of structure dependent properties of a volcanic ash soil under pasture in southern Chile. Chilean Journal of Agricultural Research 71(2), 293-303. http://dx.doi. org/10.4067/S0718-58392011000200015.

Dec, D., Dörner, J., Balocchi, O., López, I., 2012. Temporal dyna- 
mics of hydraulic and mechanical properties of an Andosol under grazing. Soil and Tillage Research 125, 44-51. https://doi.org/10.1016/j.still.2012.05.018

Demond, A., Oliviera, I., Salehzadeh, A., 1996. Packing of sands for the production of homogeneous porous media. Soil Science Society of American Journal 60, 49-53. http://dx.doi. org/10.2136/sssaj1996.03615995006000010010x

Dörner, J., Horn, R., 2006. Anisotropy of pore functions in structured Stagnic Luvisols in the weichselian moraine region in N Germany. Journal of Plant Nutrition and Soil Science 169, 213-220. https://doi.org/10.1002/ jpln.200521844

Dörner, J., Dec, D., Peng, X., Horn, R., 2009. Efecto del cambio de uso en la estabilidad de la estructura y la función de los poros de un Andisol (Typic Hapludand) del sur de Chile. Revista de la Ciencia del Suelo y Nutrición Vegetal 9(3), 190-209. http://dx.doi.org/10.4067/S071827912009000300003

Dörner, J., Dec, D., Horn, R., Peng, X., 2010. Effect of land use change on the dynamic behaviour of structural properties of an Andisol in southern Chile under saturated and insaturated hydraulic conditions. Geoderma 159, 189197. https://doi.org/10.1016/j.geoderma.2010.07.011

Dörner, J., Dec, D., Zúñiga, F., Sandoval, P., Horn, R., 2011. Effect of the land use change on Andosol's pore functions and their functional resilience after mechanical and hydraulic stress. Soil and Tillage Research 115-116, 71-79. https://doi.org/10.1016/j.still.2011.07.002

Dörner, J., Dec, D., Feest, E., Vásquez, N., Díaz, M., 2012. Dynamics of soil structure and pore functions of a volcanic ash soil under tillage. Soil and Tillage Research 125, 5260. https://doi.org/10.1016/j.still.2012.05.019

Dörner, J., Zúñiga, F., López, I., 2013. Short-term effects of different pasture improvement treatments on the physical quality of an andisol. Journal of Soil Science and Plant Nutrition 13(2), 381-399. https://doi.org/10.4067/ S0718-95162013005000031

Dörner, J., Huertas, J., Cuevas, J.G., Leiva, C., Paulino, L., Arumí, J.L., 2015. Water content dynamics in a volcanic ash soil slope in southern Chile. Journal of Plant Nutrition and Soil Science 178, 693-702. https://doi.org/10.1002/ jpln.201500112

Drewry, J., 2006. Natural recovery of soil physical properties from treading damage of pastoral soil in New Zealand and Australia: A review. Agriculture, Ecosystems and Environment 114(2-4), 159-169. https://doi. org/10.1016/j.agee.2005.11.028

Ellies, A., 1988. Mechanical consolidation in volcanic ash soils, in: Drescher, J., Horn, R., De Boodt, M. (Eds.), Impact of Water and External Forces on Soil Structure. Catena, Supplement 11, Catena Verlag, Cremlingen, pp. 87-92.

Ellies, A., Grez, R., Ramírez, C., 1997. La conductividad hidráulica en fase saturada como herramienta para el diagnóstico de la estructura del suelo. Agro Sur 25(1), 51-56.

Fujikawa, T., Miyazaki, T., 2005. Effect of bulk density ans soil type on the gas diffusion coefficient in repacked and undisturbed soils. Soil Science 170(11), 892-901. https:// doi.org/10.1097/01.ss.0000196771.53574.79

Food and Agriculture Organization of the United Nations (FAO), 2006. World Reference Base for Soil Resources (WRB). 2nd ed. World Soil Resources Reports No. 103, Rome.
González-Reyes, Á., Muñoz, A., 2013. Cambios en la precipitación de la ciudad de Valdivia (Chile) durante los últimos 150 años. Bosque 34(2), 191-200.. https://doi. org/10.4067/S0717-92002013000200008

Greenwood, K., McKenzie, B., 2001. Grazing effects on soil physical properties and the consequences for pastures: a review. Australian Journal of Experimental Agriculture 41(8), 1231-1250. https://doi.org/10.1071/EA00102

Greenwood, K., MacLeod, D., Hutchinson, K., 1997. Long-term stocking rate effects on soil physical properties. Australian Journal of Experimental Agriculture 37(4), 413419. https://doi.org/10.1071/EA96131

Hodgson, J., Illius, A., 1996. The Ecology and Managements of Grazing Systems. CAB International, Wallingford, UK.

Horn, R., Baumgartl, R., 2002. Dynamic properties of soils, in: Warrick, A. (Ed.), Soil physics companion. CRC press, Florida, USA, pp. 17-43.

Horn, R., Way, T., Rostek, G., 2003. Effect of repeated tractor wheeling on stress/strain properties and consequences on physical properties in structured arable soils. Soil and Tillage Research 73(1-2), 101-106. https://doi. org/10.1016/S0167-1987(03)00103-X

Horn, R., Smucker, A., 2005. Structure formation and its consequences for gas and water transport in unsaturated arable and forest soils. Soil and Tillage Research 82, 5-14. https://doi.org/10.1016/j.still.2005.01.002

Horn, R., Fleige, H., 2009. Risk assessment of subsoil compaction for arable soils in Northwest Germany at farm scale. Soil and Tillage Research 102(2), 201-208. https://doi. org/10.1016/j.still.2008.07.015

Hoyos, N., Comerford, N., 2005. Land use and landscape effects on aggregate stability and total carbon of Andisols from Colombian Andes. Geoderma 129(3-4), 268278. https://doi.org/10.1016/j.geoderma.2005.01.002

Ivelic-Sáez, J., Zúñiga, F., Valle, S., López, I., Dec, D., Dörner, J., 2015. Functional resistance and resilience of the pore system of an andisol exposed to different strategies of pasture improvement under sheep grazing. Journal of Soil Science and Plant Nutrition, 15(3), 663-679. https://doi.org/10.4067/S0718-95162015005000045

Keller, T., Arvidsson, J., Dawidowski, A., Koolen, R., 2004. Soil precompression stress: II. A comparison of different compaction tests and stress-displacement behavior of the soil during wheeling. Soil and Tillage Research 77(1), 97-108. https://doi.org/10.1016/j.still.2003.11.003

Krümmelbein, J., Peth, S., Horn, R., 2008. Determination of pre-compression stress of a variously grazed steppe soil under static and cyclic loading. Soil and Tillage Research 99(2), 139-148. https://doi.org/10.1016/j. still.2008.01.008

Lal, R., 1994. Sustainable land use systems and soil resilience, in: Greenland, D., Szabolcs, I. (Eds.), Soil Resilience and Sustainable Land Use. CAB International. Wallingford, pp. 41-67.

Matus, F., Amigo, X., Kristiansen, S., 2006. Aluminium stabilization controls organic carbon levels in Chilean volcanic soils. Geoderma 132, 158-168. https://doi. org/10.1016/j.geoderma.2005.05.005

Menneer, J., Ledgard, S., Mclay, C., Silvester, W., 2004. The impact of grazing animal on $\mathrm{N}_{2}$ fixation in legume-based pastures and management options for improvement. 
Advance in Agronomy 83, 181-241.

Mosaddeghi, M., Koolen, A., Hajabbasi, M., Hemmat, A., Keller, T., 2007. Suitability of pre-compression stress as the real critical stress of unsaturated agricultural soils. Biosystem Engineering 98(1), 90-101. https://doi. org/10.1016/j.biosystemseng.2007.03.006

Peng, X., Horn, R., Smucker, A., 2007. Pore shrinkage dependency of inorganic and organic soils on wetting and drying cycles. Soil Science Society of American Journal 71, 1095-1104. https://doi.org/10.2136/sssaj2006.0156

Peth, S., Horn, R., 2006. The mechanical behavior of structured and homogenized soil under repeated loading. Journal of Plant Nutrition and Soil Science 169(3), 401-410. https://doi.org/10.1002/jpln.200521942

Reszkowska, A., Peth, S., Peng, X., Horn, R., 2011a. Grazing effects on compressibility of Kastanozems in Inner Mongolian steppe ecosystem. Soil Science Society of American Journal 75(2), 426-433. https://doi.org/10.2136/ sssaj2010.0225

Reszkowska, A., Krümmelbein, J., Gan, L., Peth, S., Horn, R., $2011 b$. Influence of grazing on soil water and gas fluxes of two Inner Mongolian steppe ecosystems. Soil and Tillage Research 111(2), 180-189. https://doi. org/10.1016/j.still.2010.10.003

Savadogo, P., Sawadogo, L., Tiveau, D., 2007. Effects of grazing intensity and prescribed fire on soil physical and hydrological properties and pasture yield in the savanna woodlands of Burkina Faso. Agriculture, Ecosystems and Environment 118(1-4), 80-92. https://doi. org/10.1016/j.agee.2006.05.002

Schäffer, B., Attinger, W., Schulin, R., 2007. Compaction of restored soil by heavy agricultural machinery-Soil physical and mechanical aspects. Soil and Tillage Research 93(1), 28-43. https://doi.org/10.1016/j.still.2006.03.007

Teuber, N., Balocchi, O., Parga, J., 2007. Manejo del Pastoreo.
Imprenta América, Osorno, Chile.

Tobias, S., Tietje, O., 2007. Modelling expert's judgement on soil compaction to derive decision rules for soil protection-A case study from Switzerland. Soil and Tillage Research 92(1-2), 129-143. https://doi.org/10.1016/j. still.2006.02.001

Tomas, C., Domenech, A., Mira, A., Cuenca, S., 2007. Preconsolidation stress in the Vega Baja and Media areas of the River Segura (SE Spain): Causes and relationship with piezometric level changes. Engineering Geology $91(2-4)$, 135-151. https://doi.org/10.1016/j.enggeo.2007.01.006

Vásquez, N., Salazar, F., Dörner, J., 2012. Variabilidad temporal de las propiedades físico-mecánicas de un suelo derivado de cenizas volcánicas bajo labranza convencional. Agro Sur 40(3), 1-13.

Wichtmann, T., Niemunis, A., Triatafyllidis, T., 2005. Strain accumulation in sand due to cyclic loading: drained triaxial tests. Soil Dynamics and Earthquake Engineering 25, 967-979.

Wingching-Jones, R., Cabalceta-Aguilar, G., Alvarado-Hernandez, A., 2009. Impacto del pastoreo con ganado Holstein y Jersey sobre la densidad aparente de un Andisol. Agronomía Mesoamericana 20(2), 371-379. https:// doi.org/10.15517/am.v20i2.4953

Zhao, Y., Peth, S., Krümmelbein, J., Horn, R., Wang, Z., Steffens, M., Hoffmann, C., Peng, X., 2007. Spatial variability of soil properties affected by grazing intensity in Inner Mongolia grassland. Ecological Modeling 205(1-2), 241-254. https://doi.org/10.1016/j.ecolmodel.2007.02.019

Zuñiga, F., Ivelic-Sáez, J., López, I., Huygens, D., Dörner, J., 2015. Temporal dynamics of the physical quality of an Andisol under grazing system subjected to different pasture improvement strategies. Soil and Tillage Research 145, 233-241. https://doi.org/10.1016/j.still.2014.09.014 
